\title{
Factores psicosociales que influyen en el ausentismo: Evaluación de un modelo explicativo
}

\section{Work psychosocial conditions influencing on absenteeism:}

\section{Evaluation of an explicative model}

\author{
Edgar Guillermo Pulido Guerrero ${ }^{1}$, Lesby Johanna Lora Carrillo ${ }^{2}$ \\ y Luz Karine Jiménez Ruiz ${ }^{3}$
}
${ }^{1}$ Psicólogo. Especialista en Gerencia en Salud Ocupacional. Magíster en Proyectos Educativos mediados por TIC. Docente investigador en la Universidad de Santander, Campus Valledupar. Miembro del grupo de investigación Estudios Socio-Humanísticos, Categoría C de COLCIENCIAS. E-mail: edg.pulido@mail.udes.edu.co

${ }^{2}$ Psicóloga Social Comunitaria. Magíster en Teoría y Práctica de la Prosocialidad y sus Aplicaciones en la Logoterapia de la Universidad Autónoma de Barcelona. Docente investigador en la Universidad de Santander, Campus Valledupar. Miembro del grupo de investigación Estudios Socio-Humanísticos, Categoría C de COLCIENCIAS. E-mail: les.lora@mail.udes.edu.co ${ }^{3}$ Psicóloga. Especialista en Salud Ocupacional. Especialista en Gerencia de Talento Humano. Magíster en Gerencia del Talento Humano. Doctor en Gestión de la Innovación. Docente investigador de la Fundación Universitaria del Área Andina. Miembro del grupo de investigación

Pensamiento Diverso, Categoría C de COLCIENCIAS. Investigador Junior de Colciencias.

E-mail: 1jimenez43@areandina.edu.co

\author{
Empresa Palmas Becerril. \\ Municipio de Codazzi, \\ Departamento del Cesar, Colombia.
}

\section{Resumen}

El objetivo del presente estudio es evaluar un modelo explicativo del ausentismo laboral, con base en la relación entre los factores de riesgo psicosocial y la mediación de la percepción de relaciones sociales y del estrés laboral; este se conformó con base en las predicciones de modelos teóricos como Demandas-Control, Desbalance Esfuerzo-Recompensas y Apoyo Social, implícitos en el instrumento usado, así como en los antecedentes de investigación consultados. Para ello, se lleva a cabo un modelado con ecuaciones estructurales con los datos de medición de riesgo psicosocial y ausentismo hecha con 252 trabajadores de una compañía agroindustrial del departamento del Cesar en Colombia; fue aplicada la Batería de Riesgo Psicosocial de Villalobos, Vargas, Rondón y Felknor (2013a, 2013b). Con base en los indicadores de bondad de ajuste, se descartó el modelo teórico inicial, así como otros dos modelos planteados, por lo que se afirma que solo algunas formas específicas de ausentismo son determinadas por el estrés laboral producto de la conjunción de riesgo ante relaciones interpersonales y demandas laborales, combinado con riesgo proveniente de condiciones extralaborales. La inclusión de las relaciones interpersonales en el modelo es totalmente esperable con base en los hallazgos 
Pulido Guerrero, Lora Carrillo y Jiménez Ruiz 
de diferentes investigaciones precedentes. Por otro lado, la exclusión del factor control sobre el trabajo fue inesperada, pues en estudios antecedentes esta guardó mayor relación con el ausentismo que las demandas del trabajo. Se discute sobre las limitaciones del estudio y sobre la necesidad de complejizar en Colombia la investigación sobre la relación del riesgo psicosocial con otros constructos psicológicos y con las consecuencias organizacionales.

Palabras clave: ausentismo, factores de riesgo psicosocial, estrés laboral, psicología organizacional, modelos de ecuaciones estructurales

\section{Abstract}

The purpose of this study is to evaluate an explanatory model of work absenteeism, based on the relationship between psychosocial risk factors and the mediation of the perception of social relationships and work stress. This was based on the predictions of theoretical models such as Demands-Control, Unbalance Effort-Rewards and Social Support, implicit in the instrument used; as well as in the research background consulted. It is justified principally by the impact that absenteeism has on the stability of organizations and the relevance of its prediction for the Psychology of Work and Organizations. For the fulfillment of the objective, a modeling SEM is carried out with the psychosocial risk and absenteeism measurement data made with 252 workers of an agro-industrial company of the department of Cesar in Colombia; $96 \%$ of participants were men, with an average age of 35 years, the majority did not complete secondary studies, $89 \%$ were in operational positions, the majority in indefinite hiring; workers less than 6 months old had been excluded. The Psychosocial Risk Battery of Colombian researchers Villalobos, Vargas, Rondón, y Felknor (2013a, 2013b) was applied. This is one of the few validated and standardized instruments on Colombian population that measures psychosocial risk globally and conforms to the definitions legally accepted in the country regarding such occupational hazards. This battery measures workers' perception of intra-occupational risk factors, in particular, demands for work, control over work, social relations and leadership, as well as rewards and recognition for work; Likewise, it evaluates the extra-labor psychosocial risk associated with working conditions, as well as symptoms of work-related stress. The path analysis was executed using statistical software SPSS v25 and AMOS v24; the goodness-of-fit for the models was verified with indicators CMIN/ DF, CFI, TLI y RMSEA; the correlations coefficients between variables and the function "modification Indices" of AMOS was operated to specify the appropriate model to the data. Based on goodness-of-fit measures, the initial theoretical model was discarded. In a second model, the mediating role of the social relations factor is ruled out and Unjustified Absenteeism, Non-Remunerated Absenteeism and Work Accident Absenteeism were excluded, but the adjustment of the model was not adequate either. In a third model, the factors Control on Work and Rewards were also excluded, although the fourth model, that inlayed correlations between de independent variables was the one who had the best goodness of fit; so, it is affirmed that only some specific forms of absenteeism are determined by work-related stress due to the arrangement of risk before interpersonal relationships and labor demands, combined with risk from extra-labor conditions. The inclusion of interpersonal relationships in the model is fully expected based on the findings of different previous investigations. On the other hand, the exclusion of the control factor over work was unexpected, since in previous studies it was more related to absenteeism than the demands of work. It is suggested that the findings should be taken with caution, given the limitations of the research, in particular, the homogeneity of the participants and the lack of comparability with other productive 
contexts; However, the relevance of the study is sustained in the fact that it is one of the first attempts in the country that uses explanatory models to establish the effects of psychosocial risk factors on organizational outcomes. Complementing the above, the possibility of using the Psychosocial Risk Battery together with the measurement of other important organizational outcomes such as presenteeism, motivation and job satisfaction, job performance and productivity, organizational commitment, accident rate in the workplace, among others, are also considered to empirically validate various models exposed in theory or corroborated in other countries and productive sectors.

Keywords: absenteeism, psychosocial risk factors, job stress, organizational psychology, structural equation models

\section{Introducción}

Predecir y controlar el ausentismo laboral es un imperativo del campo de la psicología organizacional, que se sustenta en el impacto económico preponderante que este tiene sobre las organizaciones, así como el daño en imagen institucional (Frooman, Mendelson y Kevin Murphy, 2012; González, Peiró y Rodríguez-Molina, 2011). Los datos en Colombia de la Asociación Nacional de Empresarios $(2015,2016)$ indican que anualmente el ausentismo le cuesta a las empresas un $1.5 \%$ del valor de su nómina; de igual forma, la razón de ausencias equivale a 1.5 días por año por cada trabajador, asociadas en la mayoría de ocasiones al ausentismo por enfermedad general y por permisos.

El ausentismo sucede como un comportamiento planeado y consciente, a veces, o darse también por motivos involuntarios o de fuerza mayor (Riggio, 2013). La primera modalidad requiere para la empresa una eliminación completa; aun así, el ausentismo involuntario también es susceptible de ser reducido, toda vez que los problemas de salud pueden ser consecuencia del estrés laboral. Por ello, autores como Kinicki y Fugate (2018) califican al ausentismo laboral como un efecto o consecuencia de la interacción entre los estresores laborales y el afrontamiento psicológico del individuo, que implican un nivel particular de estrés laboral.

La interacción de los factores psicosociales con el estrés, sobre todo en lo tocante a demandas y exigencias del trabajo y el nivel de control y autonomía, se ve mediada por el apoyo social(Orth-Gomér, 2012). En este orden de ideas, el apoyo social parece ser un factor muy relevante: existen estudios que muestran que, entre mejor percepción de la relación con el líder, menor ausentismo laboral y, paradójicamente, entre mejor percepción de relación con colegas y pares, mayor ausentismo (Dello Russo, Miraglia, Borgogni y Johns, 2013). De acuerdo con la teoría dialéctica de Halbesleben, Whitman y Crawford (2014), tanto el ausentismo como el presentismo son estrategias usadas por los trabajadores para reducir la tensión en la relación entre jefe y subordinado. Así, el ausentismo sería usado por el trabajador en ciertas circunstancias: cuando este deniega o rechaza la conexión, cercanía o predictibilidad respecto a su jefe; cuando es aceptable la autonomía para el jefe en una situación en que haya polarización y contradicción, ya sea temporalmente o en ciertas tareas o contextos, o incluso intercambiando ausentismo y presentismo dependiendo del contexto; y también puede usar el ausentismo para reducir la polarización y el conflicto con el jefe, o por el contrario, para reconocer una dialéctica irreconciliable con su superior.

Cabe anotar que los estudios parecen sugerir mayor asociación entre el ausentismo y el control sobre el trabajo, pero no tanto así con las demandas del trabajo. Por ejemplo, en un metaanálisis llevado a cabo por Molina (2010), en el que se buscaba precisamente evidenciar los efectos del modelo demandas-control, se halló que el riesgo de sufrir episodios de ausentismo ante percepción de bajo control sobre el trabajo es estadísticamente significativo; el autor señala que 
los hallazgos anteriores en relación con las demandas laborales son inconsistentes, y los datos obtenidos por él no logran soportar la idea de que ante mayor demanda del trabajo, mayor probabilidad de tener episodios de ausencia laboral.

Se realizó una breve revisión de literatura científica, en donde se reportan varios estudios que han evaluado modelos explicativos del ausentismo; para su desarrollo, se consultó en bases de datos de revistas indexadas, en particular, Scopus, ScienceDirect, EBSCO y Proquest. Fueron usadas como palabras clave los términos absenteeism/ausentismo, psychosocial factors/factores psicosociales, job/trabajo y stress/estrés; como criterio de selección, se escogieron estudios de alcance explicativo. En tiempo, esa búsqueda estuvo limitada a artículos de la presente década, aunque se incluyó una investigación de 2006 debido a su pertinencia.

De acuerdo a lo hallado, en Japón, por ejemplo, Saijo et al. (2017) abordaron a más de 2.500 trabajadores estatales para determinar el rol mediador el apoyo social entre demandas-control por un lado, y ausentismo-presentismo laboral, por otro. A partir de regresión logística y negativa binomial llegaron a la conclusión de que el modelo demandas-control-apoyo social se asocia más al presentismo, aunque el alto apoyo social implicó un efecto protector ante el ausentismo.

Un estudio más amplio, adelantado con datos de 31 países europeos con casi 30 mil trabajadores, fue desarrollado por Niedhammer, Chastang, Sultan-Taieb, Vermeylen y Parent-Thirion (2013). Buscaban examinar un modelo del ausentismo por enfermedad con base en factores de riesgo psicosocial conocidos, así como conceptualmente emergentes. En el modelo corroborado, se encuentra que las altas demandas psicológicas, la discriminación y bullying, la baja probabilidad de promoción laboral, y el desbalance trabajo-vida para ambos sexos, son factores de riesgo para la ocurrencia del ausentismo.

Otro estudio amplio, ejecutado por Addae,
Johns y Boies (2013) con datos recolectados en más de 1.500 trabajadores de nueve países de Norteamérica, Asia y África, corroboró mediante modelamiento de ecuaciones estructurales la influencia de variables como centralización en el trabajo, locus de control, policronividad, preferencia por la diferenciación de rol de género, y percepción de apoyo social, sobre el ausentismo y la legitimación de ausentismo; así mismo, los modelos implicaban comparar los resultados entre países. Las personas con locus de control externo, policrónicos, y que defienden la diferenciación de roles de género, son más indulgentes respecto al ausentismo, mientras que las personas con centralidad laboral y percepción de apoyo social son menos indulgentes; el locus de control interno y el buen apoyo social explican el ausentismo auto-reportado. No obstante, la relación entre esas variables varía de país en país.

En Europa, particularmente en Bélgica, Janssens et al. (2014) obtuvieron una muestra de más de 3.000 trabajadores de empresas de administración pública para estudiar el impacto de factores de riesgo psicosocial en ausentismo de tiempo largo debido a salud mental $(\mathrm{MH})$ y a desórdenes músculo-esqueléticos (MSD). Mediante modelos de regresión múltiple, encontraron que la percepción de recompensa se asocia con menor riesgo ante ausentismo por $\mathrm{MH}$, mientras que altos niveles de control del trabajo y altas demandas de trabajo se asocian con menor riesgo de ausentismo por MSD, y por último, la percepción de bullying se asoció con el ausentismo debido a MH tanto como debido a MSD.

En una investigación hecha por Hanebuth, Meinel y Fischer (2006) con más de 1.500 trabajadores alemanes del sector manufacturero, se buscó evaluar un modelo explicativo del ausentismo a partir de variables como percepción y conducta en salud, características del trabajo y variables demográficas. El principal predictor hallado del ausentismo fue la calidad de vida asociada a salud física. 
De igual forma, la percepción de liderazgo y de apoyo social se asoció a ausentismo de término largo. Concluyen estos autores que la varianza del ausentismo no se puede explicar sin la percepción de salud y sin el apoyo social. Otra relación relevante sucedió entre desbalance esfuerzo-recompensa y ausentismo de corto plazo; por su parte, las demandas del trabajo y el control no estuvieron relacionados.

En Latinoamérica son menos frecuentes las experiencias de evaluación de modelos explicativos. En una investigación hecha en Brasil, Duarte, Mara y De Lima (2016) abordaron a 635 docentes de colegios públicos, con el propósito de determinar la influencia de factores personales, políticos, económicos, culturales y sociales en el ausentismo de profesores, mediado por la satisfacción laboral; usaron análisis factorial exploratorio y confirmatorio. Sus resultados confirman que el estilo de liderazgo, la remuneración, las condiciones de trabajo, la demanda laboral y los factores sociales interfieren en el ausentismo docente; para el caso, los factores con mayor peso fueron las demandas del trabajo y el tema social e interpersonal.

En Colombia existe un escenario en que se presenta poca investigación correlacional y casi nula explicativa, en torno a los factores de riesgo psicosocial, así como respecto a sus múltiples posibles consecuencias para las organizaciones (Pulido, 2015). Al hacer la pesquisa sobre relación entre factores psicosociales y ausentismo, se encuentran limitados estudios. En Cali, Guzmán (2017) abordó a 70 trabajadores de una industria licorera en una investigación descriptiva que buscaba comparar el riesgo psicosocial y el ausentismo según modalidad de contrato; encontrando que los trabajadores contratados a término fijo tienen un poco más de riesgo psicosocial y los trabajadores a término indefinido presentan mayor ausentismo. No obstante, el investigador no sugiere relaciones entre factores psicosociales y ausentismo. Algo similar sucede con la reciente investigación de Ruiz,
Castro y Cañón (2018), donde se caracterizan ambas variables, pero no se correlacionan ni se hace análisis explicativo.

La única investigación consultada que sí evaluó modelos explicativos del ausentismo, fue llevada a cabo por Restrepo y Salgado (2013). Usaron los datos de la encuesta de hogares de 2006 del Departamento Administrativo Nacional de Estadísticas, que tiene más de 24 mil participantes. Encontraron que las mujeres, las personas casadas, quienes tienen contrato verbal, quienes tienen menor nivel educativo y quienes trabajan en empresas pequeñas tienden a ausentarse más de forma significativa.

Para el presente estudio, se tiene como propósito evaluar, como modelo inicial, aquel que se muestra en la Figura 1, para el cual se considera que el apoyo social es una variable intermedia entre los demás factores psicosociales intralaborales y el estrés laboral, y a su vez, el estrés laboral media la relación entre los riesgos intralaborales y el riesgo extralaboral con el ausentismo. Se enfatizó en el posible rol mediador del apoyo social en general, dada la importancia que muestran estas variables en modelos teóricos y antecedentes arriba expuestos. En cuanto a impacto, se pretende generar evidencia en el contexto laboral colombiano acerca de la conexión entre el riesgo psicosocial y el ausentismo, definiendo una posible ruta o forma de corroborar las relaciones e influencias que señalan los modelos de mayor peso en la explicación del estrés laboral y sus efectos dentro de la psicología organizacional y el campo de la salud ocupacional, mediante métodos como el modelamiento estructural, que ha venido a tener gran acogida en diversas áreas de la psicología.

Se considera relevante la realización del presente estudio por dos razones: (1) el modelamiento de ecuaciones estructurales ha empezado a tener gran popularidad en la investigación psicológica dada su capacidad de dar explicaciones más holísticas y generales de los fenómenos de interés (Karimi y 
Meyer, 2014); y (2) en Colombia la investigación sobre factores psicosociales se ha centrado en alcances descriptivos y correlacionales y pocos estudios han asociado este fenómeno a efectos ocupacionales y organi- zacionales (Pulido, 2015), por lo que es relevante avanzar en estudios explicativos y que demuestren las consecuencias de la interacción del trabajador con riesgos ocasionados por la inserción en el mundo laboral.

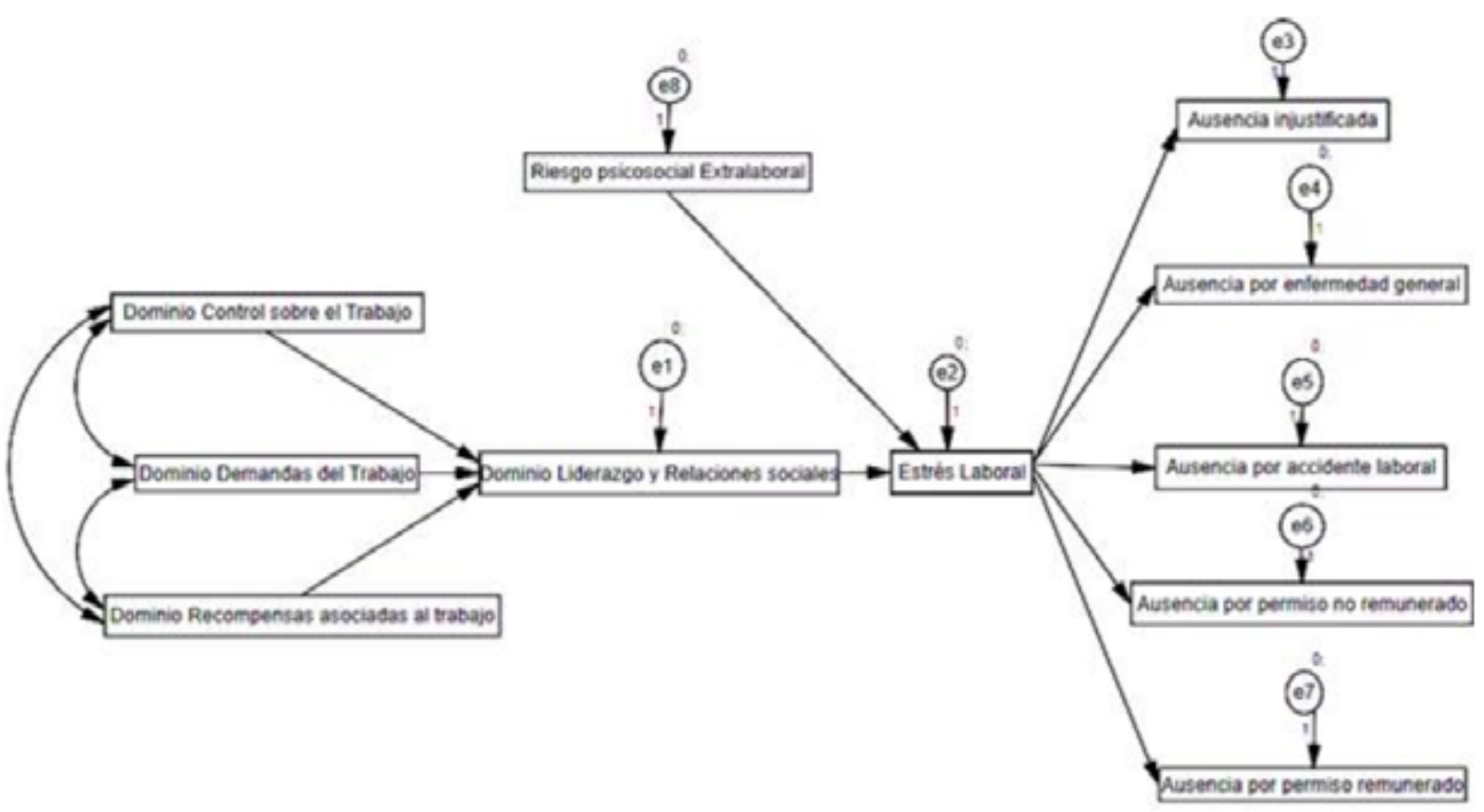

Figura 1. Modelo hipotético de la investigación. Fuente: Elaboración propia.

\section{Metodología}

\section{Participantes}

Se incluyeron a los 252 trabajadores de una empresa del sector agroindustrial productora de aceite de palma, ubicada en el municipio de Codazzi, departamento del Cesar, Colombia. El $96 \%$ de participantes eran hombres. La media de edad se ubicó en 35 años, con un mínimo de 19 años y un máximo de 63. El 58 $\%$ no había culminado los estudios de secundaria y el $26 \%$ solo contaba con bachillerato. El $89 \%$ eran trabajadores operativos, el $47 \%$ eran contratados a término indefinido y el 32 $\%$ con contrato temporal menor a un año. Se aplicó la batería a todos los trabajadores de la organización, por tanto, se hizo una investigación censal. Su usó como criterio de exclu- sión una antigüedad en la empresa menor a 6 meses.

\section{Instrumentos}

Se aplicó la Batería de Riesgo Psicosocial de Villalobos, Vargas, Rondón y Felknor (2013a, 2013b). Esta batería tiene dos formas, A y B, la primera aplicable a cargos de jefatura, técnicos/tecnológicos y profesionales, y la segunda aplicable a cargos operativos. En relación a factores psicosociales intralaborales, la batería mide liderazgo y relaciones sociales, control sobre el trabajo, demandas laborales y recompensa, que presentan alphas de Cronbach de $.948, .828, .827$ y .794, respectivamente. Sobre los factores extralaborales, mide la percepción ante condiciones fuera del trabajo, tales como disposición de tiempos, 
relaciones familiares, relaciones interpersonales, situación económica, vivienda y entorno, desplazamiento, que en el estudio de su validación evidenciaron valores de alpha entre .944 y .957. Por último, la Escala de Estrés Laboral de la batería mide el reporte de síntomas fisiológicos, de comportamiento social, intelectuales y psicoemocionales, y obtuvo un alpha de .851 .

La validez de constructo se corroboró mediante un análisis factorial de factores principales y rotación promax; de igual manera, se encontraron correlaciones significativas entre los dominios y dimensiones, así como entre dimensiones y riesgo psicosocial intra o extralaboral, según correspondiese. Asimismo, estos autores hicieron validez de criterio al medir correlación con el instrumento SF-36V. Ese proceso de validación y estandarización fue hecho con una muestra representativa aleatoria de 2.360 trabajadores colombianos.

Para la evaluación de ausentismo, se tuvo acceso a la base de datos consolidada con las ausencias por trabajador, que clasifica en los siguientes tipos: por enfermedad general (AEG), por ausencia injustificada (AI), por accidente laboral (AAT), por permiso remunerado (APR), por permiso no remunerado (APN), por suspensión y por licencias. Las dos últimas categorías fueron excluidas del análisis, al no haber lógica de dependencia de la conducta del propio trabajador.

\section{Procedimiento}

Se realizó un acercamiento a talento humano y bienestar de la organización, donde se establecieron los parámetros de la aplicación en consonancia con las directrices de la Resolución 2646 de 2008. Los instrumentos fueron aplicados en la jornada de trabajo por psicólogos con licencia en Salud y Seguridad en el Trabajo; en algunos casos, debido a la baja formación escolar (el 19 \% no terminó la primaria), fue necesario hacer lectura de los ítems a los participantes. Se explicaron las condiciones de confidencialidad y uso de datos con base en Código de Ética del Psicólogo (Ley 1090 de 2006); asimismo, se hizo uso del consentimiento informado bajo recomendaciones del Colegio Colombiano de Psicólogos (2015).

\section{Análisis de datos}

El análisis estadístico de datos se hizo mediante las herramientas ofimáticas SPSS 25 y AMOS 24 de IBM. Se llevó a cabo un Path Analysis con modelamiento de ecuaciones estructurales con el método de máxima verosimilitud; este método identifica el ajuste de modelos teóricos, basado en relaciones de variables que sugieren causalidad, siendo una extensión del modelo de regresión múltiple (Pérez, Medrano y Rosas, 2013). Se llevó a cabo la verificación de la bondad de ajuste del modelo inicial y de los modelos subsecuentes mediante los indicadores CMIN/DF, CFI, TLI y RMSEA. Asimismo, se realizó una estimación de correlaciones entre los dominios medidos, que junto con los datos de los indicadores y la función Modification Indices del programa $\mathrm{AMOS}$, sirvió de base para concretar el modelo definitivo. No se usó la puntuación directa de los instrumentos de la batería, sino el puntaje con el factor de corrección que se usa en la calificación, según las instrucciones del manual del Ministerio de Protección Social (2010).

\section{Resultados}

Para la evaluación del modelo, debe considerarse que todas las variables incluidas eran variables observadas, salvo las estimaciones de error. La Tabla 1 muestra los estadísticos descriptivos de las variables observadas. 
Tabla 1

Estadísticos descriptivos de las variables observadas.

\begin{tabular}{ccccc}
\hline Variable & $\boldsymbol{M}$ & $\boldsymbol{D T}$ & Asimetría* $^{*}$ & Curtosis** \\
\hline Liderazgo y relaciones sociales & 10.99 & 12.08 & 1.418 & 2.205 \\
Control & 19.41 & 13.22 & .639 & .024 \\
Demandas & 29.02 & 7.63 & -.055 & -.212 \\
Recompensa & 14.79 & 15.59 & 1.57 & 3.18 \\
Riesgo extralaboral & 11.51 & 8.57 & .920 & .802 \\
Estrés & 13.19 & 11.21 & 1.13 & 1.13 \\
AEG & 6.11 & 10.92 & 4.25 & 26.26 \\
AI & .79 & 1.53 & 3.72 & 20.2 \\
AAT & 1.79 & 5.58 & 7.22 & 71.51 \\
APR & .58 & 1.12 & 2.41 & 6.27 \\
APN & 1.54 & 1.58 & 1.58 & 3.46 \\
\hline
\end{tabular}

Fuente: Elaboración propia. $\mathrm{N}=252 .{ }^{*}$ Error $=.151 .{ }^{* *}$ Error $=.302$.

El modelo formulado inicialmente no tuvo evidencia de bondad de ajuste, tal y como lo indican los datos de la Tabla 2. Por ende, se llevó a cabo un análisis de correlaciones bivariadas con $r$ de Pearson. Al revisar la matriz de correlaciones (Tabla 3 ), se verifica que el estrés laboral solo tiene correlaciones significativas con ausentismo por enfermedad general y ausentismo por permiso remunerado. Con esta información, y con los hallazgos de valores de regresión y correlación de la evaluación del modelo original, se propuso un segundo modelo en el cual se descartaba el papel de variable intermedia de relaciones sociales entre los demás dominios y el estrés, y se elimina al ausentismo injustificado, ausentismo por permiso no remunerado y ausentismo por accidente de trabajo. Si bien los indicadores del modelo 2 mejoraron respecto al modelo original, aún distan de ser óptimos, como puede verse en la Tabla
2. Basándose nuevamente en la matriz de correlaciones y en los valores de regresión obtenidos en el modelo 2, se descartan las variables dominio control sobre el trabajo y dominio recompensas, que tenían $r$ de Pearson menores a .3 y valores de regresión cercanos a 0 ; no obstante, este tercer modelo resultante, donde no hay correlación entre los factores psicosociales, tampoco muestra bondad de ajuste, aunque acerca mucho más los indicadores a lo esperado. Por último, se evalúa un cuarto modelo que sí acoge la correlación entre riesgo psicosocial extralaboral, liderazgo y relaciones sociales y demandas del trabajo, así como la regresión de estas con respecto al estrés laboral, además de la influencia del estrés laboral sobre las ausencias por enfermedad general y por permisos remunerados; como se evidencia en la Tabla 2, este modelo $\mathrm{n}^{\circ} 4$ muestra indicadores óptimos de bondad de ajuste. 
Tabla 2

Indicadores de bondad de ajuste de los modelos evaluados.

\begin{tabular}{cccccccc}
\hline Modelo & Chi $^{2}$ & Sig. & CMINDF & CFI & TLI & RMSEA & PNFI \\
\hline Modelo 1 & 246.35 & .000 & 5.866 & .570 & .437 & .137 & .409 \\
Modelo 2 & 129.111 & .000 & 8.607 & .719 & .475 & .172 & .376 \\
Modelo 3 & 86.601 & .000 & 8.660 & .631 & .446 & .172 & .407 \\
Modelo 4 & 6.625 & .469 & .946 & 1.000 & 1.004 & .000 & .453 \\
\hline
\end{tabular}

Fuente: Elaboración propia. $\mathrm{N}=252$

Tabla 3

Matriz de correlaciones de las variables medidas.

\begin{tabular}{lcccccccccccc}
\hline Variable & $\mathbf{1}$ & $\mathbf{2}$ & $\mathbf{3}$ & $\mathbf{4}$ & $\mathbf{5}$ & $\mathbf{6}$ & $\mathbf{7}$ & $\mathbf{8}$ & $\mathbf{9}$ & $\mathbf{1 0}$ & $\mathbf{1 1}$ \\
\hline $\begin{array}{l}\text { 1. Liderazgo } \\
\text { y relaciones } \\
\text { sociales }\end{array}$ & 1 & $.453^{* *}$ & $.196^{* *}$ & $.481^{* *}$ & $.402^{* *}$ & $.313^{* *}$ & -.004 & .027 & -.024 & -.066 & $.133^{*}$ \\
$\begin{array}{l}\text { 2. Control } \\
\text { 3. Demandas }\end{array}$ & 1 & .067 & $.472^{* *}$ & $.333^{* *}$ & $.155^{*}$ & .050 & -.054 & -.002 & -.055 & .050 \\
$\begin{array}{l}\text { 4.Recom- } \\
\text { pensa }\end{array}$ & & 1 & $.163^{* *}$ & $.348^{* *}$ & $.402^{* *}$ & $-.149^{*}$ & .006 & .024 & -.063 & $.171^{* *}$ \\
5.Riesgo \\
extralaboral
\end{tabular}

Fuente: Elaboración propia. $\mathrm{N}=252 .{ }^{*} \mathrm{p}<.05 .{ }^{* *} \mathrm{p}<.01$.

La Figura 2 muestra los datos estandarizados del modelo 4. En este se evidencia correlaciones estadísticamente significativas entre dos de los factores intralaborales, Relaciones sociales y Liderazgo por un lado, y Demandas del trabajo por el otro, entre ellas y respecto al Riesgo psicosocial extralaboral. Por otro lado, los pesos de regresión de cada una de esas tres variables independientes respecto al estrés laboral fueron significativos, casi todos por debajo de .001 de valor $p$; la relación menos fuerte fue la correspondiente a Liderazgo y relaciones sociales, aunque el valor de $p$ fue de .024. Lo mismo sucede en los pesos de regresión del estrés sobre las dos únicas formas de ausentismo incluidas, por enfermedad general y por permisos remunerados, donde el valor $p$ más alto fue de .003 . 


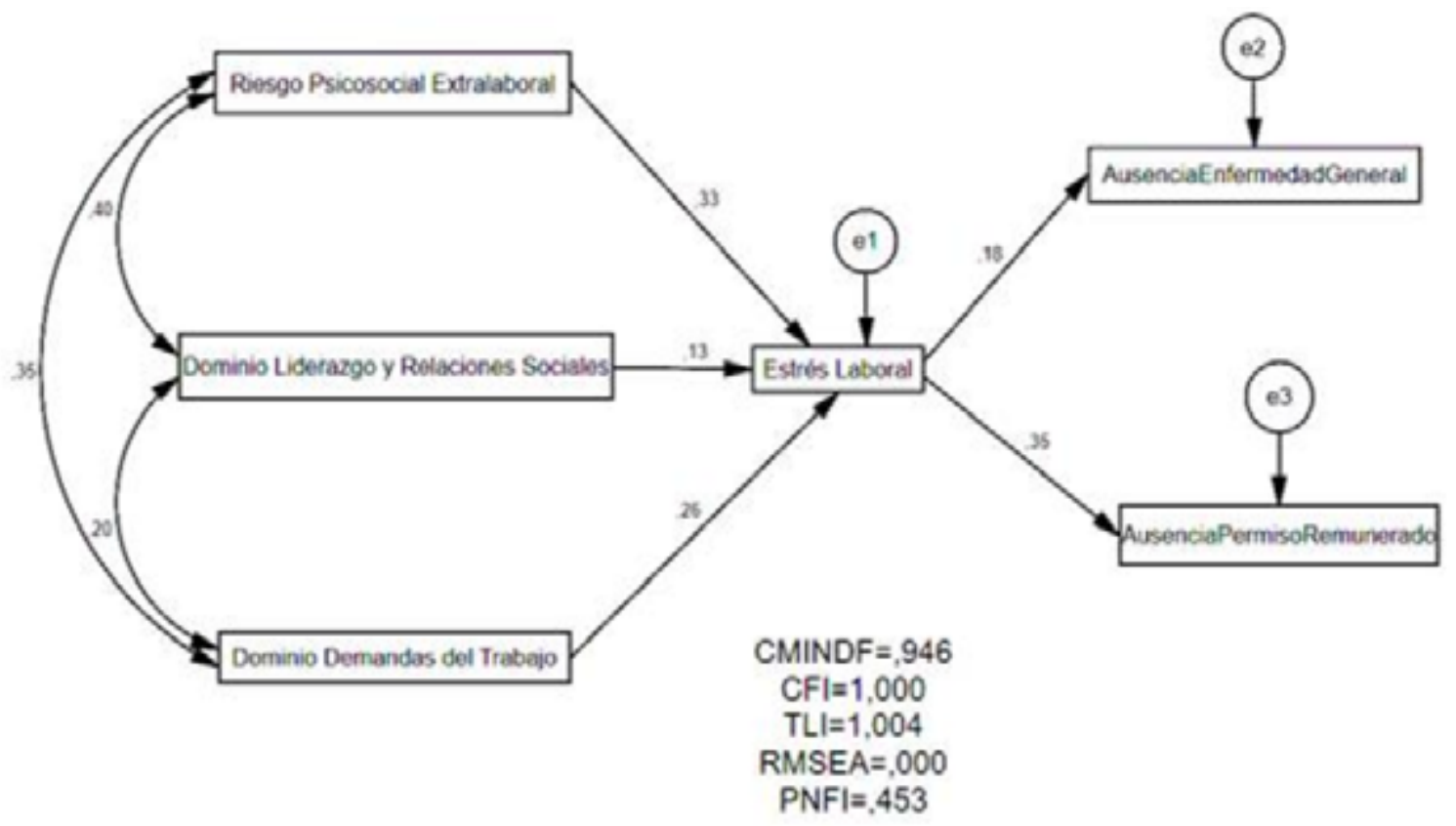

Figura 2. Modelo de ecuaciones estructurales con bondad de ajuste para la explicación del ausentismo laboral $(\mathrm{n}=252)$. Fuente: Elaboración propia.

Por último, la Tabla 4 muestra los efectos directos, indirectos y totales de cada variable observada. Debido a la estructura del modelo resultante, las variables independientes no tuvieron efecto directo sobre las medidas de ausentismo, sino solo indirectamente a través de su influencia en el estrés.

Tabla 4

Efectos directos (D), indirectos (I) y totales (T) estandarizados de las variables del modelo 3.

\begin{tabular}{cccc}
\hline Variable & $\mathbf{4}$ & $\mathbf{5}$ & $\mathbf{6}$ \\
\hline 1. Liderazgo y relaciones & $\mathrm{T}=.122$ & $\mathrm{~T}=.046$ & $\mathrm{~T}=.023$ \\
sociales & $\mathrm{D}=.129$ & $\mathrm{D}=.000$ & $\mathrm{D}=.000$ \\
& $\mathrm{I}=.000$ & $\mathrm{I}=.046$ & $\mathrm{I}=.023$ \\
& $\mathrm{~T}=.261$ & $\mathrm{~T}=.093$ & $\mathrm{~T}=.047$ \\
2. Demandas & $\mathrm{D}=.261$ & $\mathrm{D}=.000$ & $\mathrm{D}=.000$ \\
& $\mathrm{I}=.000$ & $\mathrm{I}=.093$ & $\mathrm{I}=.047$ \\
& $\mathrm{~T}=.330$ & $\mathrm{~T}=.060$ & $\mathrm{~T}=.117$ \\
3. Riesgo extralaboral & $\mathrm{D}=.330$ & $\mathrm{D}=.000$ & $\mathrm{D}=.000$ \\
& $\mathrm{I}=.000$ & $\mathrm{I}=.060$ & $\mathrm{I}=.117$ \\
& & $\mathrm{~T}=.181$ & $\mathrm{~T}=.354$ \\
4. Estrés & & $\mathrm{D}=.181$ & $\mathrm{D}=.354$ \\
& & $\mathrm{I}=.000$ & $\mathrm{I}=.000$ \\
5. AEG & & & \\
6. APR & & & \\
\hline
\end{tabular}

Fuente: Elaboración propia. $\mathrm{N}=252$ 


\section{Discusión}

Los datos demuestran que el modelo originalmente planteado no era explicativo de los resultados de las mediciones y posibles relaciones de los diferentes factores psicosociales, estrés y ausentismo. Se logró ajustar un modelo específico que cumple con los criterios de bondad de ajuste. No obstante, este modelo implicó omitir de la explicación a las variables control sobre el trabajo y recompensa, y en cuanto a factores intralaborales, basarse en demandas del trabajo y relaciones sociales y liderazgo. Es un hallazgo parcialmente inesperado, toda vez que en la literatura la evidencia de la influencia del control sobre el ausentismo es más sólida que aquella sobre la influencia de las demandas laborales en esa consecuencia organizacional (Molina, 2010); aun así, se revisaron antecedentes como el de Duarte et al. (2016), donde las demandas de trabajo jugaron un rol preponderante en explicar el ausentismo.

La presencia de la variable relaciones sociales y lideragzo en el modelo corroborado era esperable. Investigaciones revisadas como las de Saijo et al. (2017), Addae et al. (2013), Niedhammer et al. (2013), Duarte et al. (2016) o Hanebuth et al. (2006) ponen de manifiesto la importancia del apoyo social, y resultados como los de Janssens et al. (2014) demuestran que fenómenos contrarios al apoyo social, como el bullying, conllevan mayor ausentismo. Por su parte, los efectos de factores extralaborales en el ausentismo se han investigado muchas veces con base en el concepto de conflicto trabajo-familia como mediador, aunque algunas investigaciones, como la desarrollada por Vignoli, Guglielmi, Bonfiglioli y Violante (2016) no han soportado esa hipótesis. En el caso del presente estudio, los datos incluyen en el modelo a esta variable, aún por encima de algunos factores intralaborales.

Los factores psicosociales hasta aquí mencionados tienen la particularidad de presentar efectos directos nulos respecto de cualquier forma de ausentismo. Los efectos indirectos sobre el ausentismo por enfermedad general y por permiso remunerado son bajos y siempre mediados por el estrés laboral. En cambio, el peso de la regresión de estos factores sobre el estrés, y subsecuentemente, del estrés sobre estas formas de ausencia, son ambos significativos. También hay una consistente correlación entre los dos factores intralaborales del modelo entre ellos y con el riesgo extralaboral; más aún, el modelo parece sostenerse mucho en la evidencia de esas correlaciones, puesto que el modelo 3 , el cual no incluye las estimaciones de correlación de esos factores, no tiene bondad de ajuste, pero al disponer esa situación de correlación, pasa a ser óptimo según los indicadores. Puede plantearse entonces que el ausentismo, ya sea por enfermedad general, o por permisos remunerados, es determinado por el estrés laboral producto de la conjunción de riesgo ante relaciones interpersonales y demandas laborales, combinado con riesgo proveniente de condiciones extralaborales.

El hallazgo al que se concluye debe ser tomado con suma cautela, principalmente por las características de la muestra. Una cualidad de varios de los antecedentes consultados es el uso de participantes provenientes no solo de diferentes empresas, sino incluso de diferentes regiones y países. Se reconoce entonces que la validez externa de los resultados propios se ve afectada por la relativa homogeneidad entre participantes, producto de pertenecer a un mismo contexto. No obstante, el estudio desarrollado viene a ser uno de los primeros intentos de evaluar modelos explicativos que incluye factores psicosociales y consecuencias organizacionales en el país; como ya se expuso desde el análisis hecho por Pulido (2015), esa es una limitación seria en el estudio de los factores de riesgo psicosocial en Colombia. De igual forma, algunos autores han llamado la atención sobre la tendencia de la salud ocupacional en centrarse en la seguridad y prevención de accidentes para reducir el ausentismo, ignorando los efectos que 
también tiene la exposición a riesgo psicosocial sobre las ausencias en el trabajo (Jiménez, 2015).

Por tanto, se afirma que la presente investigación es un aliciente y puede denotar rutas y fomentar ideas respecto a cómo usar la metodología científica para tener un panorama más amplio sobre los efectos en las organizaciones que tienen las problemáticas de bienestar laboral y estresores asociados al trabajo. En particular, se pretende demostrar la utilidad y aplicabilidad del modelamiento estructural para estudiar las diferentes dinámicas en las que se involucran los factores psicosociales en el trabajo en su rol, como determinante tanto del bienestar individual como del propio funcionamiento de la organización bajo la consideración de los outputs organizacionales.

Desde la propuesta ejecutada, se quiere hacer una invitación a los investigadores nacionales preocupados en temas como el riesgo psicosocial, la productividad y la sostenibilidad de las empresas basadas en los recursos humanos, en profundizar en la explicación de las complejas relaciones entre los factores implicados. Más aún, teniendo a disposición una herramienta de aplicación en investigación tan preponderante como la Batería de Evaluación de Factores de Riesgo psicosocial, que es a la fecha el único instrumento validado en Colombia para hacer la medición respectiva, y que muchas veces es usada solo para caracterizar el riesgo, pero no para relacionarlo con otros constructos psicológicos o con las consecuencias organizacionales que tanto importan a los empresarios y el sector productivo.

En este orden de ideas, se plantea que en el futuro la medición realizada con la Batería de Riesgo Psicosocial, que acoge los principales factores del trabajo reconocidos teóricamente como estresores, puede usarse en modelos que expliquen una diversidad de fenómenos de interés para la psicología de las organizaciones y del trabajo, entre ellos el presentismo, la motivación y satisfacción laboral, el desempeño laboral y la productiv- idad, el compromiso organizacional, la accidentalidad en el sitio de trabajo, entre otros. De igual manera, se requiere la ratificación de los hallazgos presentados en contextos laborales diferentes, tanto a nivel de contexto geográfico y cultural, como en otros sectores económicos y productivos; en este caso, es recomendable realizar estudios comparativos, con muestras diversas y con una cantidad de participantes más amplia.

\section{Referencias bibliográficas}

Addae, H. M., Johns, G. y Boies, K. (2013).

The legitimacy of absenteeism from work: a nine nation exploratory study. Cross Cultural Management: An International Journal, 20(3), 402-428. https://doi.org/10.1108/CCM-052012-0040

Asociación Nacional de Empresarios. (2015). Encuesta Ausentismo, Incapacidades y Restricciones Médicas 2015. http://www.andi.com. co/SBCB/Paginas/Divulgación-.aspx

Asociación Nacional de Empresarios. (2016). Informe de seguimiento sobre salud y estabilidad en el empleo. http:/www.andi.com.co/ Uploads/(2) Primer informe de seguimiento sobre salud y estabilidad en el empleo 2016 23052018.pdf

Colegio Colombiano de Psicólogos. (2015). Diseño y evaluación de una lista de chequeo para la elaboración del consentimiento informado en el ejercicio profesional de la psicología en Colombia. https://issuu.com/colpsic/ docs/consentimiento_informado_colpsic

Dello Russo, S., Miraglia, M., Borgogni, L. y Johns, G. (2013). How time and perceptions of social context shape employee absenteeism trajectories. Journal of Vocational Behavior, 83(2), 209-217. https://doi.org/10.1016/J. JVB.2013.03.005

Duarte, V., Mara, S. y De Lima, M. (2016). An explanatory model of teacher absenteeism: analysis and proposition. In A. Goel (Ed.), Values: An organizational resource (pp. 138-146). http://www.fumec.br/sites/ isswov/wp-content/uploads/2016/07/Book 
ISSWOV2016.pdf

Frooman, J., Mendelson, M. B. y Kevin Murphy, J. (2012). Transformational and passive avoidant leadership as determinants of absenteeism. Leadership y Organization Development Journal, 33(5), 447-463. https://doi. org/10.1108/01437731211241247

González, M. G., Peiró, J. M. y Rodríguez-Molina, I. (2011). El absentismo laboral: Antecedentes, consecuencias y estrategias de mejora. https://books.google.es/books?id=GRyOBriyVJ4Cydq=efectos+del+ausentismo+laboralyl$\mathrm{r}=\mathrm{yhl}=$ esysource $=\mathrm{gbs} \_$navlinks_s

Guzmán, D. F. (2017). Factores de riesgo psicosocial y ausentismo laboral de una industria licorera por modalidad contractual (Universidad del Valle). http://bibliotecadigital.univalle. edu.co/xmlui/bitstream/handle/10893/12557/ CB-0574770.pdf? sequence $=1$

Halbesleben, J. R. B., Whitman, M. V. y Crawford, W. S. (2014). A dialectical theory of the decision to go to work: Bringing together absenteeism and presenteeism. Human Resource Management Review, 24(2), 177-192. https:// doi.org/10.1016/j.hrmr.2013.09.001

Hanebuth, D., Meinel, M. y Fischer, J. E. (2006). Health-related quality of life, psychosocial work conditions, and absenteeism in an industrial sample of blue- and white-collar employees: a comparison of potential predictors. Journal of Occupational and Environmental Medicine, 48(1), 28-37. http://www.ncbi.nlm. nih.gov/pubmed/16404207

Janssens, H., Clays, E., De Clercq, B., Casini, A., De Bacquer, D., Kittel, F. y Braeckman, L. (2014). The relation between psychosocial risk factors and cause-specific long-term sickness absence. The European Journal of Public Health, 24(3), 428-433. https://doi. org/10.1093/eurpub/cku009

Jiménez, L. K. (2015). Psycho-labor risk factors in the mining sect. Tesis Psicológica, 10(1), 116-130. http://www.redalyc.org/articulo. oa?id=139044651009

Karimi, L. y Meyer, D. (2014). Structural Equation Modeling in Psychology: The History, Development and Current Challenges. Interna- tional Journal of Psychological Studies, 6(4). https://doi.org/10.5539/ijps.v6n4p123

Kinicki, A. y Fugate, M. (2018). Organizational behavior. A practical, problem-solving approach. New York: McGraw Hill.

Ministerio de Protección Social. (2010). Batería de Instrumentos para la Evaluación de Factores de Riesgo Psicosocial. http://www.fondoriesgoslaborales.gov.co/documents/Publicaciones/ Estudios/Bateria-riesgo-psicosocial-1.pdf

Molina, J. M. (2010). Metanálisis: Relación entre factores psicosociales en el trabajo y absentismo laboral. Medicina y Seguridad Del Trabajo, 56(220), 200-219. http://scielo. isciii.es/scielo.php?script=sci_arttextypi$\mathrm{d}=\mathrm{S} 0465-546 \mathrm{X} 2010000300003$

Niedhammer, I., Chastang, J.-F., Sultan-Taieb, H., Vermeylen, G. y Parent-Thirion, A. (2013). Psychosocial work factors and sickness absence in 31 countries in Europe. The European Journal of Public Health, 23(4), 622-629. https://doi.org/10.1093/eurpub/cks124

Orth-Gomér, K. (2012). El Apoyo Social: un modelo interactivo del estrés. In Organización Internacional del Trabajo (Ed.), Enciclopedia de Saludy Seguridad en el Trabajo. http://www. insht.es/portal/site/Insht/menuitem.1fla3bc79ab34c578c2e8884060961ca/?vgnextoid=a981ceffc39a5110VgnVCM100000dc0ca8c0RCRDyvgnextchannel=9f164a7f8a651110VgnVCM100000dc0ca8c0RCRD

Pérez, E., Medrano, L. A. y Rosas, J. S. (2013). El Path Analysis: conceptos básicos y ejemplos de aplicación. Revista Argentina de Ciencias Del Comportamiento, 5(1), 52-66. https://doi. org/10.30882/1852.4206.V5.N1.5160

Pulido, E. G. (2015). Investigaciones en factores psicosociales en el trabajo en Colombia: una revisión. Inclusión y Desarrollo, 3(1), 83-95.

https://doi.org/10.26620/uniminuto.inclusion.3.1.2016.83-95

Restrepo,C.y Salgado,E.(2013). Types of contracts and worker absenteeism in Colombia. Journal of Business Research, 66(3), 401-408. https:// doi.org/10.1016/J.JBUSRES.2012.04.006

Riggio, R. E. (2013). Introduction to Industrial/ Organizational Psychology (Sixth Edit). New 
Jersey: Pearson.

Ruiz, A. Y., Castro, K. G. y Cañón, A. (2018).

Factores de riesgo psicosocial y ausentismo laboral en los colaboradores de una empresa de fundición de metales no ferrosos, Bogotá 2016 - 2018 (Universidad Distrital Francisco José de Caldas). http://repository.udistrital. edu.co/handle/11349/14622

Saijo, Y., Yoshioka, E., Nakagi, Y., Kawanishi, Y., Hanley, S. J. B. y Yoshida, T. (2017). Social support and its interrelationships with demandcontrol model factors on presenteeism and absenteeism in Japanese civil servants. International Archives of Occupational and Environmental Health, 90(6), 539-553. https://doi. org/10.1007/s00420-017-1218-y

Vignoli, M., Guglielmi, D., Bonfiglioli, R. y Violante, F. S. (2016). How job demands affect absenteeism? The mediating role of workfamily conflict and exhaustion. International Archives of Occupational and Environmental Health, 89(1), 23-31. https://doi.org/10.1007/ s00420-015-1048-8

Villalobos, G. H., Vargas, A. M., Rondón, M. A. y Felknor, S. A. (2013a). Design of psychosocial factors questionnaires: A systematic measurement approach. American Journal of Industrial Medicine, 56(1), 100-110. https://doi. org/10.1002/ajim.22071

Villalobos, G. H., Vargas, A. M., Rondón, M. A., y Felknor, S. A. (2013b). Validation of new psychosocial factors questionnaires: A Colombian national study. American Journal of Industrial Medicine, 56(1), 111-123. https:// doi.org/10.1002/ajim.22070

Recibido: 23 de mayo de 2019 Aceptado: 22 de octubre de 2020 